\title{
A novel noninvasive assessment of hepatic venous pressure gradient and portal pressure computed from computed tomography angiography
}

\author{
Xiaolong $\mathrm{Qi}^{1}$, Fangyu Zhou ${ }^{2}$, Huijie $\mathrm{LV}^{3}$, Huisong Chen ${ }^{1}$, Weiguo Xu ${ }^{4}$, Surong Xing ${ }^{4}$, Fei Wang ${ }^{1}$, \\ Changqing Yang ${ }^{1}$
}

\author{
${ }^{1}$ Division of Gastroenterology and Hepatology, Tongji University School of Medicine, \\ Tongji Hospital, Shanghai, China \\ ${ }^{2}$ Tongji University School of Medicine, Shanghai, China \\ ${ }^{3}$ Division of Mathematics, Tongji University, Shanghai, China \\ ${ }^{4}$ Division of Radiology, Tongji Hospital, Tongji University School of Medicine, \\ Shanghai, China
}

Submitted: 8 October 2012

Accepted: 13 December 2012

Arch Med Sci 2014; 10, 5: 1052-1054

DOI: 10.5114/aoms.2014.46224

Copyright $\odot 2014$ Termedia \& Banach

Cirrhosis is a severe and common digestive disease worldwide and is associated with a poor outcome. Portal hypertension (PHT) is a frequently encountered complication in cirrhotic patients [1-3]. Therefore, evaluation of portal pressure (PP) is urgently needed for the grading and staging of PHT. Direct measurement of PP is rarely performed due to significant invasiveness and potential complications. Hepatic venous pressure gradient (HVPG) is currently treated as the "gold standard" [1-4]. However, the invasiveness and technical difficulty greatly restrict its repeatable application.

An index computed from computed tomography angiography (CTA) has recently demonstrated its diagnostic value compared with the "gold standard" $[5,6]$. For instance, fractional flow reserve from coronary CTA showed good performance in the diagnosis of functional coronary stenosis in a multicenter randomized clinical controlled trial on 159 vessels in 103 patients [6]. On the basis of our previous study of noninvasive measurement of coronary stenosis by fractional flow reserve [7], this study, for the first time, introduces a novel noninvasive assessment of HVPG $\left(H V P G_{n i}\right)$ and PP $\left(P_{n i}\right)$ computed from three dimensional (3D) hepatic portal venous models reconstructed from CTA to diagnose the severity of PHT in cirrhotic patients.

The patient enrolled in the pilot study was a 50-year-old man who showed visible clinical manifestations of PHT, such as ascites and splenomegaly, and was diagnosed with cirrhosis in the decompensated stage. Approval was obtained from the ethical committees of Tongji Hospital and the participant gave written informed consent in advance.

The CTA was performed with multi-detector CT scanners (GE LightSpeed Ultra, 120 kV, $220 \mathrm{~mA}$ ). Original images were spilt into thin layers and exported into imaging control software MIMICS10.0. 3D hepatic portal venous models were reconstructed from surrounding tissues by different CT values, which were then meshed with 3D Flotran elements in the software ANSYS11.0. Given boundary conditions including average velocity of hepatic venous and portal venous flow were measured by color Doppler ultrasound (CDUS) (Philips iU22 x MATRIX Ultrasound System,

\author{
Corresponding author: \\ Prof. Changqing Yang MD, \\ $\mathrm{PhD}$ \\ Division of Gastroenterology \\ and Hepatology \\ Tongji University \\ School of Medicine \\ Tongji Hospital \\ 389 Xin Cun Road \\ 200065 Shanghai, China \\ Phone: +86 2166111076 \\ Fax: +862166111604 \\ E-mail: cqyang@tongji.edu.cn
}




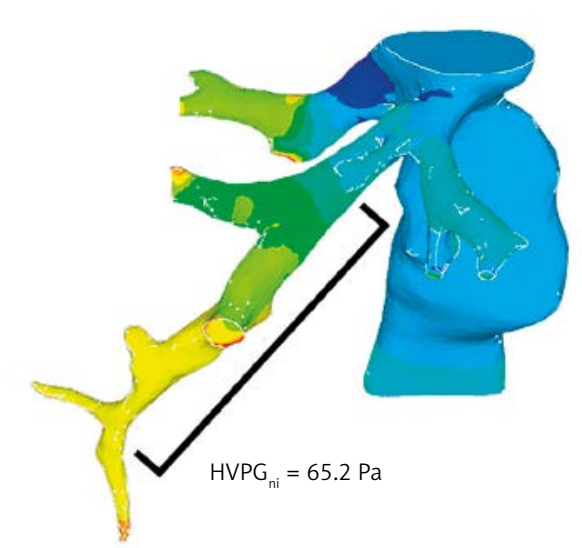

Pressure $[\mathrm{Pa}]$

\section{0}

690

Figure 1. Noninvasive assessment of hepatic venous pressure gradient in decompensated cirrhosis. Hepatic venous pressure gradient was calculated in vitro with the value of $65.2 \mathrm{~Pa}$

convex array probe (5-2). Afterwards, finite element analysis and computational fluid dynamics were applied to compute the pressure distribution in the hepatic portal vein in vitro.

Clinical data of the decompensated cirrhotic patient were calculated to test the feasibility and accuracy of the novel noninvasive assessment with the clinical diagnosis, CTA and CDUS as reference standards. The CTA images showed that the diameter of the portal vein was $1.6 \mathrm{~cm}$ while the normal diameter is $\leq 1.3 \mathrm{~cm}[2,3]$. Besides, the average velocity of portal venous flow was $12.5 \mathrm{~cm} / \mathrm{s}$, which was obviously slower than $22.62 \mathrm{~cm} / \mathrm{s}$ in normal patients [1, 3, 4] and indicated severe PHT. According to the calculation by the novel assessment, $\mathrm{HVPG}_{\mathrm{n}}$ and $\mathrm{PP}_{\mathrm{ni}}$ were computed as 65.2 Pa (Figure 1) and 3535.0 Pa (Figure 2), which generally corresponded to the diagnosis of PHT. Furthermore, the pressure and velocity distribution of different sections could be simultaneously obtained.

The PHT is known as a frequent complication of cirrhosis worldwide which significantly reduces the patient's quality of life. The HVPG is currently considered as the "gold standard" to identify PHT. However, shortcomings, such as invasiveness and technical difficulty, limit its diagnostic worth greatly.

In the pilot study, finite element analysis and computational fluid dynamics over the 3D hepatic portal venous model were applied, which potentially developed a novel noninvasive assessment of $\mathrm{HVPG}_{n i}$ and $\mathrm{PP}_{\mathrm{ni}}$ to evaluate the severity of PHT. Besides, the novel assessment was successfully applied and the diagnostic performance of $\mathrm{HVPG}_{n}$

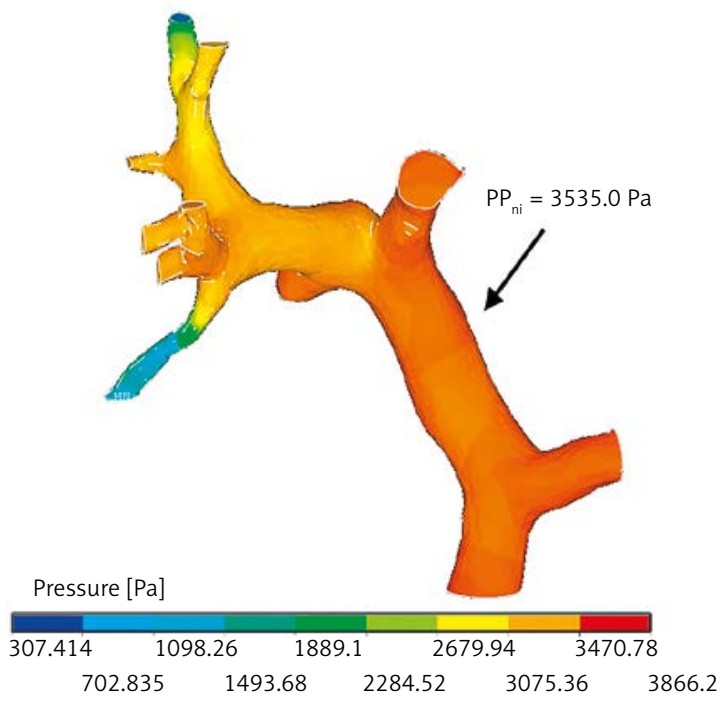

Figure 2. Noninvasive assessment of portal pressure in decompensated cirrhosis. Portal pressure was quantified in vitro as $3535.0 \mathrm{~Pa}$ and was generally consistent with the clinical diagnosis of portal hypertension

and $\mathrm{PP}_{\mathrm{ni}}$ was overall consistent with the clinical diagnosis, CTA and CDUS.

This study introduces, for the first time, a novel noninvasive assessment of HVPG ${ }_{n i}$ and $\mathrm{PP}_{\mathrm{ni}}$ computed from hepatic portal venous models, which might be a potential diagnostic tool to evaluate the severity of PHT, grading of cirrhosis and choice of treatment in the future. However, large randomized clinical controlled trials evaluated by both the novel assessment and invasive "gold standard" are needed before the application from bench to bedside.

\section{Acknowledgments}

This study was supported by the National Natural Science Foundation of China (81070343); Outstanding Academic Leader of Shanghai (08×D14045); Research Training of Tongji University (1500219050).

\section{References}

1. Thabut D, Moreau R, Lebrec D. Noninvasive assessment of portal hypertension in patients with cirrhosis. Hepatology 2011; 53: 683-94.

2. Bosch J, Abraldes JG, Berzigotti A, García-Pagan JC. The clinical use of HVPG measurements in chronic liver disease. Nat Rev Gastroenterol Hepatol 2009; 6: 573-82.

3. Zhang L, Yin J, Duan Y, Yang Y, Yuan L, Cao T. Assessment of intrahepatic blood flow by Doppler ultrasonography: relationship between the hepatic vein, portal vein, hepatic artery and portal pressure measured intraoperatively in patients with portal hypertension. BMC Gastroenterol 2011; 11: 84.

4. Vizzutti F, Arena U, Rega L, Pinzani M. Noninvasive diagnosis of portal hypertension in cirrhotic patients. Gastroenterol Clin Bio 2008; 32: 80-7. 
5. Koplay M, Kantarci M. Common hepatic artery arising from the aorta - demonstration with multidetector CT angiography and its clinical importance. Arch Med Sci 2011; 7: 176-7.

6. Koo BK, Erglis A, Doh JH, et al. Diagnosis of ischemiacausing coronary stenosis by noninvasive fractional flow reserve computed from coronary computed tomographic angiograms results from the prospective multicenter discover-flow study. J Am Coll Cardiol 2011; 58: 1989-97.

7. Qi X, Lv H, Zhou F, et al. A novel noninvasive method for measuring fractional flow reserve through three-dimensional modeling. Arch Med Sci 2013; 9: 581-3. 